\title{
FOODLIT-PRO: conceptual and empirical development of the food literacy wheel
}

\author{
Raquel Rosas ${ }^{a}$, Filipa Pimenta ${ }^{a}$, Isabel Leal ${ }^{a}$ and Ralf Schwarzer ${ }^{b, c}$ \\ ${ }^{a}$ WJCR - William James Center for Research, ISPA - Instituto Universitário, Lisboa, Portugal; ${ }^{b}$ Department of Psychology, Freie \\ Universität Berlin, Berlin, Germany; 'Department of Clinical, Health, and Rehabilitation Psychology, SWPS University of Social Sciences \\ and Humanities, Wroclaw, Poland
}

\begin{abstract}
Pursuing food systems' sustainability is crucial. Given the risk constituted by unhealthy diets, scarce research on food-related adjacent fields, and inconsistency across food literacy conceptualizations, this study aims to explore the constructs' definition and develop a conceptual and empirical framework of food literacy. A quantitative approach was taken on previously obtained qualitative outcomes from 30 interviews with experts from food-related fields. Food literacy was defined by a four-dimension model: Cooking Skills, Preserve and Analyse, Choice and Acquisition, Search and Plan. The framework Food Literacy Wheel integrates the construct definition, food literacy determinants (Internal, External) and influential factors (Nutritional, Psychological, Health, Learning Contexts, Policy, Industry, Sustainability, Social and Cultural). Allowing a broader perspective of food literacy within major food systems, this study contributes with new insights for future instruments and interventions, paving the way to develop/implement food literacy-related multi-sectorial and multilevel actions.
\end{abstract}

\section{ARTICLE HISTORY}

Received 21 February 2020

Revised 25 April 2020

Accepted 27 April 2020

\section{KEYWORDS}

Food literacy; definition; mixed-methods; framework; conceptual; empirical

\section{Introduction}

Safeguarding nutritional nourishment and food security worldwide have been permanent cross-cutting concerns for major food-related agencies, being integrated as objectives in most action programmes (FAO et al. 2019). The population growth across the globe, the volatile development of world economy, and the unstable climate circumstances faced nowadays have led to major shifts within the ambit of food and nutrition (FAO et al. 2019; The World Bank 2019). Particularly, impacting not only food infrastructures and processes (meaning anything from production to processing, packaging, distribution, consumption, and disposal) but also causing significant effects on challenges such as food (in)security, general nutrition, and global health (FAO 2017; FAO 2019).

Acknowledging that poor diets constitute a greater risk to morbidity and mortality than the risks of unprotected sex, alcohol, tobacco and drug use combined (Global Panel on Agriculture and Food Systems for Nutrition 2016; Willett et al. 2019), it is also crucial to improve individuals' food-related knowledge and skills allowing for healthier food choices on the long term (Vidgen and Gallegos 2014; Willett et al. 2019). Paired with the need for sustainable food systems, the demand to enhance individuals' and communities' nutrition nourishment and consequent quality of life by improving their food-related competencies highlights the relevance of research and action within the field of food literacy (Vidgen and Gallegos 2014; Vettori et al. 2019; Willett et al. 2019). As an increasingly used construct, the meaning of food literacy is not yet consensual among researchers. Mainly characterised as a set of food-related awareness, knowledge, skills and practices, food literacy has been set to protect diet quality across one's lifespan, operating to improve nutritional health; this is ideally achieved focussing on one's relationship with food, and recognising social and cultural influences within this relation (Block et al. 2011; Desjardins et al. 2013; Vidgen and Gallegos 2014; Cullen et al. 2015; Perry et al. 2017; Slater et al. 2018; Thomas et al. 2019; Vettori et al. 2019).

Regarding empirical conceptualizations of food literacy, Vidgen and Gallegos (2014) included four domains - Plan and Manage, Select, Prepare, and Eat 
- within the comprehension of this construct, specifically concerning food-related knowledge, practical skills and specific behaviours at an individual level. In another perspective, Thomas et al. (2019) identified five categories as the conceptualisation of food literacy: Food and Nutrition Knowledge, Food Skills, Selfefficacy and Confidence, Ecologic, and Food Decisions. Within the detailed attributes belonging to these categories, the authors go beyond the stated individual level (Vidgen and Gallegos 2014) by incorporating specific social determinants of global health and including the influence of food systems on individual, societal and environmental aspects (Thomas et al. 2019). Despite being exclusively based on a scoping review, Cullen and co-authors (Cullen et al. 2015) stated not only individual skills and social determinants of health but also community aspects (particularly related to food security). These were integrated within global food systems, and their relation to general health and wellbeing enhancement were also noted (Cullen et al. 2015). Despite targeting food literacy competencies exclusively for youth transitioning to an independent living through adulthood, Slater and colleagues (Slater et al. 2018) included three domains of competencies: Functional (Confidence and Empowerment with Food), Relational (Joy and Meaning through Food), and Systems (Equity and Sustainability for Food Systems). Within these domains, the authors highlighted the individual knowledge and skills needed to have nutrition health, the emotional and cultural aspects essentials for a positive relationship with food, as well as the social, environmental and corporate features that influence food systems (Slater et al. 2018).

Significant resemblance is noted among these conceptualizations of food literacy domains and definitions, emphasising how food-related individual skills, behaviours, emotions, cultural and social aspects, and environmental features are integrated within this concept (Truman et al. 2017; Slater et al. 2018; Thomas et al. 2019). Nevertheless, (i) the absence of cultural and environmental-related aspects in the majority of these conceptualizations, (ii) the scarce exploration of psychological and emotional features, (iii) the main focus on an individual perspective, and (iv) the poor manifestation of food systems-related surrounding contexts (e.g. political and educational) reflect the inconsistency that characterises food literacy research. Thus, though already identified as crucial in order to be food literate, food-related adjacent fields (such as policy and education) are yet alienated from most theoretical frameworks (Cullen et al. 2015; Ronto et al. 2016).
Being a privilege of food literacy, the achievement of sustainability within food systems is crucial for purposes of both general health and environmental protection (Vettori et al. 2019; Willett et al. 2019). As so, and considering that food systems include much more than health and environmental consequences for the final consumer, a multi-sectorial approach is demanded to achieve transformation (International Food Policy Research Institute 2017; Bhunnoo 2019; FAO 2019; Vettori et al. 2019). Comprehensive strategies including multi-stakeholders and multilevel perspectives are necessary to concede systematic and holistic change across the diverse factors encompassed by food systems (Bhunnoo 2019; FAO 2019; Willett et al. 2019). This means that only by taking action involving agriculture, water (and other resources) usage, environmental concerns (like climate change and biodiversity loss), health issues, educational matters, economic factors, policy development, and cultural and social aspects, will it be possible to tackle challenges concerning sustainable food systems (FAO 2019; Willett et al. 2019).

Aiming to provide evidence-based research on food literacy while incorporating surrounding fields of action that may act as drivers for sustainable food systems, this team has conducted an in-depth qualitative study that explores food literacy's domains, determinants and influential factors (Rosas et al. 2019). As part of a major ongoing project designated as FOODLIT-PRO: Food Literacy Project, this study also presents a comparison of its obtained outcomes with other conceptualizations of food literacy, facilitating a clear perspective on the construct's comprehension and integration within the current literature. With outcomes partially corroborated by current research, this first study of FOODLIT-PRO identified nine domains of food literacy: Origin (e.g. knowing food origin), Safety (e.g. hygiene and safety practices), Choice and Decision (i.e. choice and decision skills), Select and Acquire (e.g. finding nutritionally equivalent foods), Plan (e.g. planning food intake ahead), Preserve (i.e. preservation skills), Prepare (i.e. preparation skills), Cook (e.g. matching ingredients), and Knowledge (i.e. declarative and procedural knowledge). As influential factors, eight categories were manifested: Nutritional (e.g. awareness of nutritional needs), Psychological (e.g. manage emotions to manage food intake), Health (i.e. food impact on health), Learning Contexts (e.g. professional support), Policy (e.g. regulation prior to consumption), Industry (e.g. marketing's influence), Sustainability (e.g. impact of food importation), Social and Cultural (e.g. evolution 
of food availability and access). Finally, Internal (e.g. prioritising food) and External (e.g. perishable and/or unreliable food-related information) determinants were also identified (Rosas et al. 2019). In addition to the innovative arrangement of food literacy's definition, this work provided for a distinct comprehension of influential factors that have particular roles within a broader food system. However, the exploration of original associations and internal structural cohesion among the new content of food literacy's domains, as well as the integration of the recently emerged food literacy's influential factors and determinants within a unique framework, are lacking.

Hence, this second study of FOODLIT-PRO intends (i) to further explore the previously identified food literacy domains (Study 1; Rosas et al. 2019) by using a quantitative methodology, aiming to determine the most suited internal structure for the construct definition, and (ii) to develop a new food literacy framework that integrates the formerly manifested influential factors and determinants (Study 1; Rosas et al. 2019), providing the contextual-related heterogeneity that is lacking within current literature.

\section{Method}

\section{Study approach and design}

Aiming to develop a conceptual model integrating the domains concerning the definition of food literacy and its interrelation with food literacy's influence factors - which were originally driven from an in-depth qualitative exploration of this construct - mixed methodologies were applied in this research (Elo and Kyngäs 2008; Costa et al. 2013). As so, this cross-sectional and exploratory study derives from an initial qualitative deductive-dominant content analysis (Hsieh and Shannon 2005; Rosas et al. 2019) which intended to explore food literacy's domains, influential factors and determinants, from the outlook of Portuguese professionals from diverse food-related fields. Posteriorly to the in-depth qualitative approach previously published (Rosas et al. 2019), a multiple correspondence analysis (MCA) was performed on the qualitatively analysed data. This quantitative approach was applied in order to (i) map and explain original associations among the manifested qualitative codes previously obtained and (ii) generate an explanatory model with these inherent associations, to develop a conceptual model that mirrors a further understanding of food literacy's definition, and including its factors of influence and determinants. Although this paper concerns particularly the findings from the quantitative approach, sufficient detail from the qualitative analysis is provided for context.

\section{Recruitment and sampling}

Potential participants were reached directly by the leading researcher and by participants' referencing (snow-ball sampling, non-probabilistic convenience sampling) between February and June 2018. Institutions were intentionally selected in order to reach a variety of organisations from across the (a) food-system and (b) other related fields -including not only (a) food production, processing, distribution, marketing, consumption, and disposing, but also (b) education, policy-making, association with human and environmental health, and sustainability. Inclusion criteria were (i) being 18 years or older, (ii) having minimum literacy and being capable of undertaking an extensive audio recorded interview, (iii) working in areas (in)directly related with food, and (iv) being responsible for one's own feeding. The criteria (iv) being responsible for one's own feeding was assessed through the presence of at least one out of the four possible items: holding responsibility over their food

(a) choice and decision, (b) selection and acquisition,

(c) preparation, and/or (d) cooking (based on the model of Vidgen and Gallegos 2014).

The sample comprehended 30 Portuguese experts (20 women and 10 men; Table 1) with ages between

Table 1. Participants' socio-demographic characteristics.

\begin{tabular}{lcc}
\hline Socio-demographic characteristics & Frequency $(\mathrm{n})$ & Percentage (\%) \\
\hline Affective-sexual relationship & 27 & \\
$\quad$ Yes & 3 & 90 \\
$\quad$ No & 1 & \\
Educational level & 2 & 3.3 \\
$\quad$ Middle school & 19 & 6.7 \\
High school & 6 & 63.3 \\
Bachelor & 2 & 20 \\
Master & & 6.7 \\
Doctorate & 29 & \\
Professional status & 1 & 96.7 \\
Active & & 3.3 \\
Unemployed & 7 & \\
Professional area & 6 & 23.3 \\
Education & 5 & 20 \\
Health & 8 & 16.7 \\
Agricultural Industry & 4 & 26.7 \\
Commercial Industry & & 13.3 \\
Food Policy & 27 & \\
Territorial area & 2 & 90 \\
$\quad$ Metropolitan area of Lisbon & 1 & 6.7 \\
Norte & & 3.3 \\
Alentejo & 5 & 16.7 \\
Annual household income & 7 & 23.3 \\
10.000 EUR or less & 12 & 40 \\
10.001 EUR-20.000 EUR & 6 & 20 \\
20.001 EUR-37.500 EUR & & \\
37.501 EUR-70.000 EUR & & \\
\hline
\end{tabular}

Note. Territorial area according to the Portuguese Nomenclature of Territorial Units for Statistical Purposes (NUTS II). 
23 and 57 years $(M=38.4 ; S D=8.60)$ from multiple food-related areas across 26 different organisations including profit and non-profit, governmental and non-governmental entities. The fields in which the professionals work were grouped into five specific areas: Education (e.g. high school and environmental education), Health (e.g. nutrition, psychology, medicine), Food Policy (e.g. development and implementation of national/international priority programmes), Agricultural Industry (e.g. production and consulting), and Commercial Industry (e.g. marketing and product innovation). Sample characteristics are described in greater detail in our previously published paper (Rosas et al. 2019).

\section{Ethical considerations}

This study was approved by the Ethics Committee of ISPA-Instituto Universitário (ref. D/002/03/2018). Information concerning the aim and methods of this research was provided to all participants; the voluntary and confidential nature of their participation and information given was guaranteed. The leading researcher also assured to all participants that their identities would not be disclosed. Additional clarification regarding the study's purpose was provided, when requested. All participants provided oral and written consent for the interview, along with a written authorisation specific for the audio recording.

\section{Data collection}

Data gathering started with the filling of a sociodemographic (e.g. education level and household income) and health-related behaviours (e.g. alcohol and tobacco consumption) questionnaire. Interviews were carried out in either the participants' employer institution or the facilities of the WJCR-William James Centre for Research; all contexts allowed for a private setting with an individual room behind closed doors. From March to June 2018, a total of 30 semistructured interviews were carried out by the leading research in person $(n=21)$ and by telephone $(n=9)$. Interviews' duration ranged form 25 to 120 minutes $(\mathrm{M}=53.65$ minutes; $\mathrm{SD}=19.88)$ and continued until data saturation was achieved, meaning that no new contents were being included or explored. Randomised revisions were carried out to verify the transcriptions' accuracy to the recorded audio.

\section{Interview protocol}

Given its complexity and description length, the protocol for the semi-structured interview was presented with full detail in this team's previously published paper (Rosas et al. 2019). Informed by published research concerning food literacy and comprehending mostly open-ended questions, the developed protocol intended to explore the domains of food literacy, as well as its influential factors and potential determinants (Vidgen and Gallegos 2014; Thomas et al. 2019). The complete protocol is currently attached in the appendices Supplementary Appendices.

\section{Data analysis}

Interviews were analysed with the MAXQDA 2018 software, and the complete process of data analysis which included initial and secondary qualitative data analyses, and data analyses verification - took place between April 2018 and January 2019. The developed categorisation matrix and its content were comprehended by (i) major categories - that is, categories that manifested the leading explored themes (for example, the domains of food literacy such as Origin, Safety, Choice and Decision, Select and Acquire, Plan); and (ii) their respective attributes - that is, the qualitative codes manifested as attributes of those categories (for example, the code planning skills and planning food intake ahead, which belong to the category Plan). Most categories and their respective attributes were theoretically informed; however, when particular text segments did not showed a proper match with the published and available food literacyrelated literature, new codes and categories inductively surface (Elo and Kyngäs 2008). Not being the primary focus of this study, the process for the qualitative analysis and the obtained results are fully described in our previously published paper (Rosas et al. 2019).

Posteriorly to the qualitative analysis, the descriptive method of MCA was performed using SPSS statistics (version 25; SPSS Inc., Chicago, IL). With an exploratory character, this multivariate statistical technique allows the mapping of existing active relations within a set of categorical variables - in this study's case, the presence/absence of the manifested qualitative attributes. Aiming to provide a summarised representation of the greatest amount of information initially contained within the variables, this technique extracts factorial dimensions that describe the juxtaposition among patterns of co-occurring responses across participants (Costa et al. 2013; Di Franco 
2016). Thus, the use of a MCA is particularly relevant and powerful when a large amount of qualitative data is collected, because this technique maximises all the qualitative content in the identification of the extracted factors and assures that the qualitative information is not sub-optimized within the data analysis (Costa et al. 2013). Criteria concerning the exploratory statistic included (a) a minimum eigenvalue of 1 for each dimension, and (b) a minimum of $5 \%$ of the total variance explained by each dimension.

\section{Strategies for trustworthiness}

Considering the significance of consistency within a qualitative coding process, the analysis of agreement among inter-raters reflects the reliability among a given description of a particular subject identified between researchers (Warrens 2014). Hence, in order to assess reliability for the qualitative analysis, two researchers independently coded three transcribed interviews using the final coding matrix. Cohen's kappa coefficient was used, considering it is adequate for nominal categories and two coders (Cohen 1960; McHugh 2012). In light of both researchers' analysis, the Cohen's kappa indicator expressed a nearly perfect agreement $(\kappa=0.82)$.

\section{Results}

\section{Data collection and emerged content}

Considering the diverse interview's duration range and to explore potential differences in the manifested content given by the participants, a Wilcoxon-MannWhitney nonparametric test was performed in order to compare the number of different qualitative codes emerged between interviews below the mean duration (equal or inferior to 53.65 minutes; group 1) and above the mean time (superior to 53.65 minutes; group 2). Within all the conducted interviews, there was no significant difference among emerged content of the two groups $(\mathrm{U}=143.50 ; \mathrm{W}=198.50 ; p=.06)$.

\section{Multiple correspondence analysis}

In order to ascertain the most suited structure for the construct's definition, a MCA was performed using the qualitative attributes that were incorporated by the domains of food literacy previously published (Rosas et al. 2019). Concerning the presence/absence of the attributes across the respondents' manifested content, these qualitative codes were treated as nominal variables. Though driven from FOODLIT-PRO's first study, rearrangements to some domains' attributes were made in order to integrate them within the present quantitative methodology.

Firstly, belonging to the domain Origin, the attributes food additives and how origin relates to food quality were merged into a singular variable. Since food additives have the function of, among others, preventing product quality impairment and maintaining its safety and nutritional value, the qualitative closeness of these codes led to a unique variable designated as additives and food quality (Food additives [WHO] 2018). Secondly, incorporated in the domain Safety, the attributes hygiene and safety practices and pesticides and herbicides were also combined. Considering that both substances for pest control and hygiene practices have crucial roles on food safety, this shared qualitative content was acknowledged by the merger of these attributes into the variable food safety practices (FAO and WHO 2019; Food safety 2019). Finally, belonging to the domain Cook, the attributes using different cooking techniques and matching cooking techniques to ingredients' nutritional value were also combined into a single variable; concerning that the latter explicitly includes the former, this overlap of content is presently embraced in the variable cooking techniques and nutritional value. Although included as a domain, both attributes of declarative and procedural Knowledge were not included as variables for the MCA given its invariability across all the respondents.

Highlighting the individuals' active role in the development of their food literacy-related knowledge, skills and behaviours, attributes that expressed intentional behavioural strategies were also added as variables to integrate the MCA (Schwarzer and Luszczynska 2015; Vaitkeviciute et al. 2015). As so, despite being acknowledged as attributes belonging to food literacy influential factors, five variables mirroring behavioural strategies that an individual could intentionally carry out in order to develop food literacy were considered (Table 2). Two of these variables constitute attributes from the influential factor Nutrition (tracking food intake and interpret nutritional labels), two were from Sustainability (buying from local/national trade, and consuming single-use food-related items), and one was from Learning Contexts (information seeking).

From the MCA analysis, a four-dimension MCA solution (with the designations Cooking Skills, Preserve and Analyse, Choice and Acquisition, and Search and Plan) was considered the most adequate output (Table 3). The dimensions presented the most significant factor loadings ranging from 0.094 to 
Table 2. Variables (attributes) included in the multiple correspondence analysis, and corresponding qualitative category concerning its relation to food literacy (domain or influential factor).

\begin{tabular}{ll}
\hline Domains & $\begin{array}{l}\text { Attributes } \\
\text { Knowiging Origin } \\
\text { Seasonality } \\
\text { Bio/Organic: Definition and Impact } \\
\text { Additives and Food Quality }\end{array}$ \\
& Food Safety Practices \\
Choice and Decision Skills \\
Choice and Decision & Selection and Acquisition Skills \\
Select and Acquire & Nutritionally Equivalents Foods \\
Plan & Planning Skills \\
& Plan Food Intake Ahead \\
Preserve & Preservation Skills \\
Prepare & Preparation Skills \\
Cook & Cooking Skills \\
& Matching Ingredients \\
& Using Recipes \\
& Cooking Motivation/Attitude \\
& Cooking Techniques and Nutritional Value \\
Influential Factors & Attributes \\
Nutrition & Tracking Food Intake \\
Interpret Nutritional Labels \\
Sustainability & Local/National Trade \\
& Single-use Food-related Items \\
Learning Contexts & Information Seeking \\
\hline
\end{tabular}

0.474, eigenvalues from 2.073 to 3.318 , and inertia from 0.094 to 0.151 . Accounting for $47.44 \%$ of the total variance, dimensions' variances ranged from 9.421\% to $15.083 \%$ and Cronbach's alpha varied from 0.54 to 0.73 . Although the commonly accepted lower limit for Cronbach's alpha is 0.70 , smaller values are acceptable for exploratory research, with literature emphasising an alpha greater than 0.50 as satisfactory for studies with 25-50 respondents (Davis 1964; Johnson and Wichern 2007).

\section{FOODLIT-PRO's food literacy wheel}

Posteriorly to the performance of the MCA, a graphic visual representation of both qualitative and quantitative outcomes was developed (Figure 1). Presenting the definition of food literacy at its core, this framework mirrors the four-dimensional quantitative structure (obtained through the MCA) which was applied to the manifested content from the first qualitative indepth study (Rosas et al. 2019). Furthermore, the Food Literacy Wheel also integrates the domain Knowledge, with two individual rings representing both declarative and procedural knowledge, applicable to any dimension of food literacy.

Subsequently to the presentation of food literacy's domains, an intentional barrier illustrated with linear points sets the beginning of categories that, despite being external to food literacy's definition, impact and are impacted by this construct. Formerly manifested and identified in the first study of FOODLIT-PRO (Rosas et al. 2019), food literacy's determinants (Internal and External) and influential factors (Nutrition, Psychological, Health, Learning Contexts, Policy, Industry, Sustainability, and Social and Cultural) are represented within separate rings and segments, respectively. Given their possible inner or outer nature, determinants are illustrated as two independent rings, representing internal and external determinants respectively. Demonstrating broader contexts comprehended by major food systems, the influential factors are illustrated as areas that actively interact with food literacy; as so, they are portrayed in the most external ring as areas of interplay with food literacy. As so, the external section of the wheel emphasises the segregation from the construct definition, highlighting the arising of contextual-related aspects by expressing features that can affect the development and enhancement of individuals' food literacy.

\section{Discussion}

\section{Multiple correspondence analysis}

This study aimed to further investigate the meaning of the construct food literacy, by exploring its domains through the employment of a quantitative methodology (multiple correspondence analysis) on previously gathered and analysed qualitative data (Rosas et al. 2019). Furthermore, given the current state of the art in the field of food literacy and its mirrored need to consolidate this concept within the function of major food systems, the development of a conceptual model integrating food literacy's definition, determinants and fields of influence was also an established goal of this paper.

With a mixed methodology, the firstly developed qualitative study gathered a total of 80 qualitative codes (mentioned by, at least, $10 \%$ of the sample) nested among 19 categories; food literacy domains comprised nine categories, determinants incorporated two categories, and influential factors integrated eight categories (Rosas et al. 2019). In the present study, a MCA was applied to food literacy domains, its respective codes and codes portraying intentional behavioural strategies; the domain Knowledge was exceptionally not comprised by the MCA since it was invariable across participants' manifested content. For this, some qualitative codes were transformed in order to be a part of this quantitative methodology. Subsequently, all codes were understood as nominal 
Table 3. Four-dimensional representation of the definition of food literacy.

\begin{tabular}{|c|c|c|c|c|c|}
\hline \multirow[b]{2}{*}{ Attributes } & \multicolumn{4}{|c|}{ Dimensions } & \multirow[b]{2}{*}{ Mean } \\
\hline & $\begin{array}{l}\text { Cooking } \\
\text { Skills }\end{array}$ & $\begin{array}{c}\text { Preserve and } \\
\text { Analyse }\end{array}$ & $\begin{array}{l}\text { Choice and } \\
\text { Acquisition }\end{array}$ & $\begin{array}{l}\text { Search and } \\
\text { Plan }\end{array}$ & \\
\hline Knowing Origin & .155 & .009 & .164 & .002 & .083 \\
\hline Seasonality & .048 & .052 & .058 & .284 & .111 \\
\hline Bio/Organic: Definition and Impact & .111 & .338 & .125 & .003 & .144 \\
\hline Additives and Food Quality & .094 & .065 & .043 & .007 & .052 \\
\hline Food Safety Practices & .065 & .197 & .063 & .075 & .100 \\
\hline Choice and Decision Skills & .000 & .021 & .317 & .014 & .088 \\
\hline Selection and Acquisition Skills & .027 & .046 & .462 & .072 & .152 \\
\hline Nutritionally Equivalents Foods & .040 & .017 & .065 & .292 & .103 \\
\hline Planning Skills & .088 & .120 & .036 & .313 & .139 \\
\hline Plan Food Intake Ahead & .188 & .203 & .027 & .227 & .161 \\
\hline Preservation Skills & .010 & .374 & .243 & .000 & .157 \\
\hline Preparation Skills & .317 & .004 & .010 & .085 & .104 \\
\hline Cooking Skills & .269 & .014 & .199 & .042 & .131 \\
\hline Matching Ingredients & .381 & .001 & .009 & .000 & .098 \\
\hline Using Recipes & .290 & .115 & .016 & .008 & .107 \\
\hline Cooking Motivation/Attitude & .403 & .085 & .005 & .036 & .132 \\
\hline Cooking Techniques and Nutritional Value & .474 & .143 & .002 & .006 & .156 \\
\hline Tracking Food Intake & .001 & .330 & .200 & .003 & .133 \\
\hline Interpret Nutritional Labels & .032 & .075 & .191 & .090 & .097 \\
\hline Local/National Trade & .139 & .261 & .039 & .168 & .152 \\
\hline Single-use Food-related Items & .016 & .014 & .158 & .131 & .080 \\
\hline Information Seeking & .170 & .083 & .046 & .214 & .128 \\
\hline Eigenvalue & 3.318 & 2.569 & 2.478 & 2.073 & 2.610 \\
\hline Inertia & .151 & .117 & .113 & .094 & .119 \\
\hline$\%$ Variance & 15.083 & 11.678 & 11.265 & 9.421 & 11.862 \\
\hline Cronbach's Alpha & .732 & .640 & .625 & .542 & - \\
\hline
\end{tabular}

Factor loadings (bold values indicate the most significant factorial weights), eigenvalues, inertia, percentage of variance and Cronbach's alphas.

variables and a MCA was performed, where a fourdimensional solution demonstrated to be the most suited outcome for the definition of food literacy.

The first dimension - designated Cooking Skills (15.08\% of total variance; $\alpha=0.73)$ - reflected the association among a total of seven codes regarding the preparation of food items ("Preparation Skills"), practical cooking competences ("Matching Ingredients", "Using Recipes", "Cooking Skills"), origin- and cooking-related impacts on food quality ("Additives and Food Quality", "Cooking Techniques and Nutritional Value"), and cooking-related psychological variables ("Cooking Motivation/Attitude"). Preparation and cooking competences have been linked with one another across not only nutrition and food literacyrelated literature but also studies referring to interventions aiming to improve one's interaction with food (Block et al. 2011; Murimi 2013; Vidgen and Gallegos 2014; Santarossa et al. 2015; Velardo 2015; Palumbo 2016; Slater and Mudryj 2016; Perry et al. 2017; Slater et al. 2018; Palumbo et al. 2019; Thomas et al. 2019; Vettori et al. 2019). In the same perspective, the feature of quality within foods was also alluded to the absence of additives in a recent study (Wijayaratne et al. 2018); however, functions of preventing product's quality deterioration and maintaining its nutritional value of origin are associated to food additives
(Food additives [WHO] 2018). Also related to food quality, it has been studied that the cooking technique applied to a particular food item affects its nutritional value (Martínez-Hernández et al. 2013; RonceroRamos et al. 2017); this supports the association represented in this dimension among these two attributes. Considering the relevance of one's relation with food in order to develop food literacy, food's preparation and cooking are set to be less about the produced meal and more about the significance of the process (Vidgen and Gallegos 2014). As so, highlighting the importance of associating psychological variables (such as motivation, confidence, and empowerment) to food-related practical skills (Thomas and Irwin 2011; Cullen et al. 2015; Krause et al. 2016), this corroborates the integration of the attribute of cooking motivation within this dimension.

The second dimension, nominated Preserve and Analyse (11.68\% of total variance; $\square=0.64$ ), aggregated five codes related to both food safety and preservation ("Preservation Skills", "Food Safety Practices", "Bio/Organic: Definition and Impact", "Local/National Trade"), and food intake ("Tracking Food Intake"). Across these variables, the analysis of food-related information demonstrates to be crucial so that associated specific behaviours - aiming for enhanced food literacy - may be developed. Given 


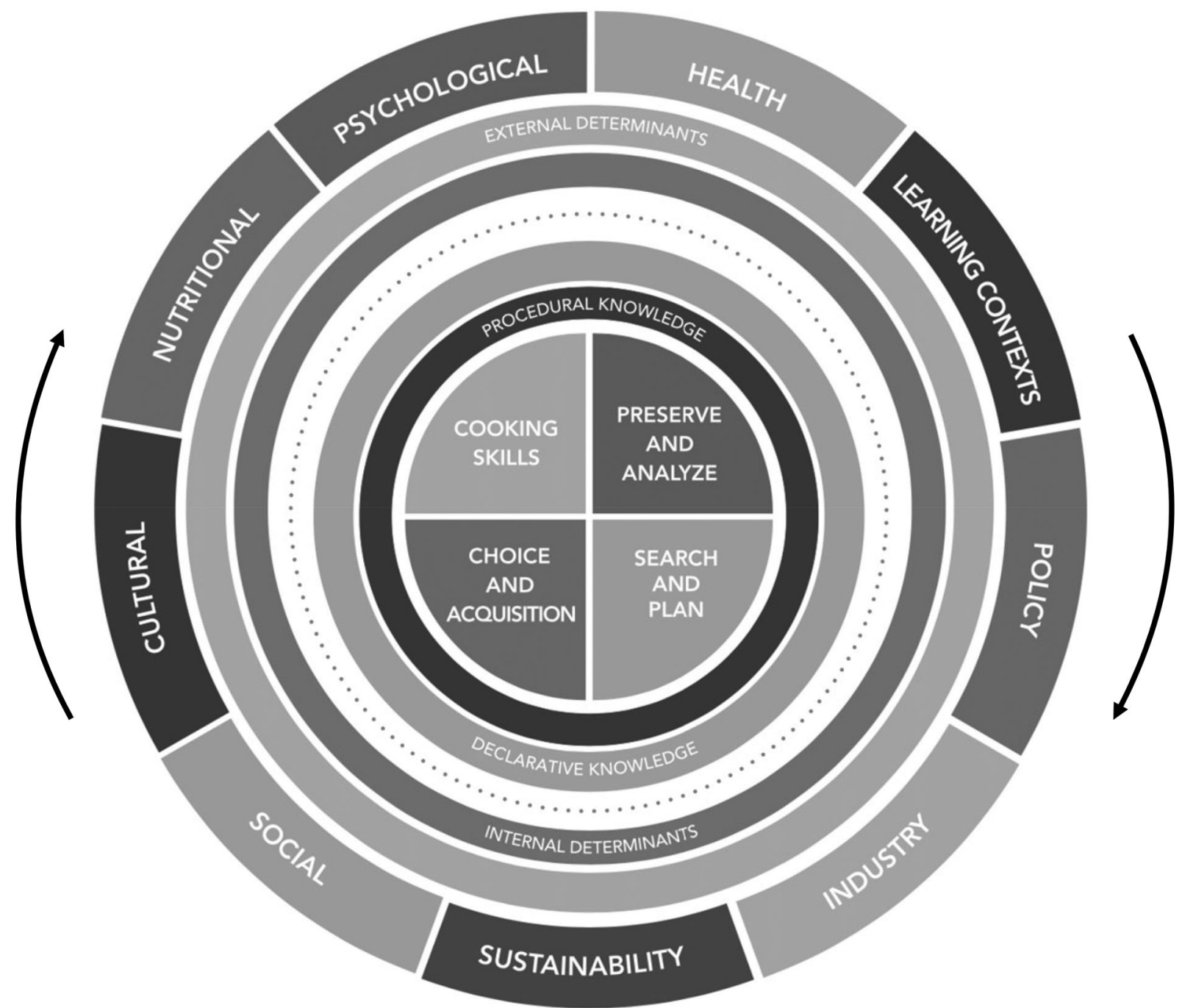

Figure 1. Food Literacy Wheel, presenting (from the inside core to the outside rings): the four-dimensional definition of food literacy (Cooking Skills, Preserve and Analyse, Search and Plan, and Choice and Acquisition), associated knowledge (procedural and declarative), its determinants (internal and external), and its influential factors.

that (i) basic preservation methods (e.g. freezing, dehydrating) and safety principles (e.g. appropriate cleaning, cooking temperature) are applied according to the food item characteristics, (ii) perceiving a food as bio/organic and as a local/national product requires the access and interpretation of origin-related information, and (iii) monitoring one's intake demands an intentional examination of regular food-related routines, it was imperative to incorporate the feature of food-related analysis on the designation of this dimension (Preserve and Analyse) (Mukhopadhyay et al. 2017; WHO 2019). Though food items' storage (belonging to "Preservation Skills") and hygienic handling (included in "Food Safety Practices") have been associated with each other in the current literature, the incoherence is significant given their understanding as part of either safety, selection competencies, knowledge, preparation skills, or general food techniques (Vidgen and Gallegos 2014; Cullen et al. 2015; Perry et al. 2017; Thomas et al. 2019; Vettori et al. 2019). Additionally to assembling these codes, the need to clarify consequences of biological/organic foods ("Bio/Organic: Definition and Impact") and the consumption of local or national foods ("Local/ National Trade") have also been stated in previous research (Merriam 2005; Sumner 2013; Durmus et al. 2018; Butcher et al. 2019). Besides the uncertainty between organic or local foods as what is more beneficial, studies also demonstrate that people associate organic products with more healthful foods and that consumers who are aware of what organic foods are, also have more knowledge about food safety practices (Sumner 2013; Durmus et al. 2018; Uhlmann et al. 2018). Regardless of this outlook, choosing local or 
national products is stated as a form of contributing to food-related sustainability, supporting local farming systems, community and economy, and reducing food-related anthropic actions over global environment (Canfora 2016; Perry et al. 2017; Powell and Wittman 2018; Willett et al. 2019). Similarly characterised as the self-awareness of what to include and restrict in one's regime, ensuring a regular food intake has been included in food literacy literature (Vidgen and Gallegos 2014; Vettori et al. 2019). As an intentional behavioural strategy, "Tracking Food Intake" was originally manifested as the monitoring of the foods and/or calories consumed (Rosas et al. 2019). However, the integration of this code within this dimension uncovers the possibility of broadening this variable for diverse content where the tracking mechanism may be applicable; as so, in spite of the initial content to be monitored referred to food or caloric intake, this assemble may be widened to focus on the quality - tracking the intake of, for example, organic and/or local products - instead of merely the quantity of the foods consumed.

The third dimension - named Choice and Acquisition (11.27\% of total variance; $\square=0.63$ ) comprehended five codes concerning features that may be considered when selecting and/or making decisions about food ("Knowing Origin", "Interpret Nutritional Labels", "Single-use Food-related Items", "Choice and Decision Skills", and "Selection and Acquisition Skills"). Accessing origin-related information and transforming it into knowledge in order to choose, decide, and acquire food items are recognised competencies across the food literacy literature (Block et al. 2011; Vidgen and Gallegos 2014; Powell and Wittman 2018; Thomas et al. 2019; Vettori et al. 2019). Similarly to choosing and acquiring foods according to its origin (for example, local versus imported foods), the use of disposable food-related items appears to be a criteria progressively taken into account for the consumer, with a particular negative emphasis on plastic-based packaging and takeout containers due to its adverse environmental and sustainability effects (Barnes et al. 2011; Lindh et al. 2016). However, though food literacy has been characterised as the understanding of the impact of food choices on - among others - environmental issues (Thomas et al. 2019), food-related plastic-based and single-use materials are yet to be linked to the construct's conceptual scope. On the contrary, reading and interpreting nutritional labels, as well as understanding basic nutritional information and guidelines, are skills linked to both nutritional and food literacy (e.g.
Vidgen and Gallegos 2014; Perry et al. 2017; Sumner 2013; Thomas et al. 2019; Vettori et al. 2019). The association of the code "Interpret Nutritional Labels" to a dimension that highlights skills of choice and decision-making as well as of food selection and acquisition reflects the already demonstrated need to interrelate this variable with other practical competencies to assess one's food literacy level/quality (Poelman et al. 2018). Likewise, both "Choice and Decision Skills" and "Selection and Acquisition Skills" are competencies constantly featured within the food literacy literature (e.g. Block et al. 2011; Vidgen and Gallegos 2014; Cullen et al. 2015; Perry et al. 2017; Wijayaratne et al. 2018; Thomas et al. 2019; Vettori et al. 2019). Recognising the complexity of these competencies and how they may not always be executed rationally, the development and/or enhancement of these skills transversally across behaviour-related interventions demonstrates to be crucial (National Academies of Sciences, Engineering, and Medicine 2016).

Finally, the fourth dimension, designated Search and Plan (9.42\% of total variance; $\alpha=0.54$ ), incorporated five codes that mirror a set of behaviours and competencies (related to "Seasonality" and "Nutritionally Equivalents Foods") linked by both the need to seek information (i.e. "Information Seeking") and the need to plan in advance (i.e. "Planning Skills" and "Plan Food Intake Ahead"). Having knowledge concerning the identification of seasonal foods (i.e. declarative knowledge, belonging to the qualitative domain Knowledge) and applying such knowledge by consuming seasonal products (i.e. procedural knowledge, also integrated in the domain Knowledge) are both contemplated in the code "Seasonality". Though scarcely represented in current food literacy frameworks (Rosas et al. 2019), seasonality has been increasingly pointed out as crucial not only for the balance of one's nutritional intake quality but also for environmentally friendly eating habits (Medina 2011; van Dooren et al. 2014; Barbosa et al. 2017; Willett et al. 2019). However, in order to have a predominantly seasonal-based food intake, knowledge about which foods are nutritionally equivalent ("Nutritionally Equivalents Foods") is necessary given the need to substitute an unavailable food item for a nutritionally similar option. Within the current literature, the content that most closely resembles to this nutritional-related matters concerns knowledge about nutrition and its application to food choices (e.g. Block et al. 2011; Vidgen and Gallegos 2014; Cullen et al. 2015; Perry et al. 2017; Thomas et al. 2019; 
Vettori et al. 2019). The association of the code "Information Seeking" emphasises how consumers' active search for information, particularly nutritionaland seasonal-related, is crucial to have awareness and understanding of seasonal foods so that one could not only acquire declarative knowledge on the matter but also to develop procedural knowledge and further food-related behaviours (Wilkins 2002; Macdiarmid 2014). Concerning procedural knowledge and practical competencies, planning aptitudes have significantly been stated in the food literacy literature and in some of the instruments developed to date (e.g. Vidgen and Gallegos 2014; Cullen et al. 2015; Velardo 2015; Krause et al. 2016; Begley et al. 2018; Poelman et al. 2018; Wickham and Carbone 2018; Amouzandeh et al. 2019). Both codes integrated within this dimension that relate to planning competencies (i.e. "Planning Skills" and "Plan Food Intake Ahead") emphasise the need to prepare in advance one's seasonal-based and nutritionally balanced food regime; planning not only what to buy according to the season and how to nutritionally replace unavailable foods, but also what to ingest so that nutritional needs are met and fulfilled, are examples of how planning competencies may be linked to the other variables aggregated in this fourth dimension.

\section{FOODLIT-PRO's food literacy wheel}

With its core representing the definition of food literacy obtained through mixed-methodologies (four-dimension model developed with qualitative deductive-dominant content analysis and multiple correspondence analysis), the design of FOODLIT-PRO's Food Literacy Wheel (Figure 1) is meant to be interpreted from the inside out. As so, the invariable domain Knowledge is graphically illustrated by the rings (expressing both procedural and declarative knowledge, respectively) following the model at the centre; this particular visual representation of the domain Knowledge intends to express the invariability of this code as part of the food literacy definition by continuously embracing all four core dimensions. In a broader understanding, this aims to disclose that the food literacy definition includes both procedural and declarative knowledge across all its dimensions and features.

Succeeding to its core, the Food Literacy Wheel presents a clear ring deliberately placed between the definition of the construct and the manifested determinants (internal and external, respectively) of food literacy, previously identified in FOODLIT-PRO's first study (Rosas et al. 2019). Stating (i) food as a priority, (ii) convenience and practicality, (iii) time and (iv) financial management, (v) previous food-related habits, and (vi) innate and (vii) learned flavour preferences as "Internal Determinants", and (a) information access, (b) perishable/unreliable information, (c) lack of food access (food security), (d) family dynamic/ identity, and (e) professionals' unpreparedness on food-related expertise as "External Determinants", this external graphic section of the wheel portrays what may limit or enhance one's food literacy (Rosas et al. 2019; Vettori et al. 2019).

Though scarcely explored and absent from most frameworks, food literacy's intersection with other food systems-related surrounding contexts (such as economic, political, cultural, and environmental features) has been recognised as being essential for the understanding of food literacy beyond the individual level (Bellotti 2010; Pendergast et al. 2011). The most external ring of the Food Literacy Wheel intends not only to express this extension of food literacy's wider contextual surroundings but also to portray fields of influence that may impact individuals' and communities' food literacy. Thus, the previously analysed and identified Influential Factors (Rosas et al. 2019) are represented in segmented parts of a unique more distal ring: (1) Nutritional, (2) Psychological, (3) Health, (4) Learning Contexts, (5) Policy, (6) Industry, (7) Sustainability, (8) Social and (9) Cultural. As fields of interplay with food literacy, the intent of contemplating the nine factors separately emphasises the dynamic rotation allowed by the wheel; this rotation was thought and created to represent how each Factor of Influence may impact and/or be impacted by any of the four dimensions of food literacy. For example, "Display of Products Information" belonging to the Policy Factor (graphically placed on the right side of the wheel) may impact individuals' and wider populations' selection and acquisition skills (competencies that integrate the dimension Choice and Acquisition, located on the left side of the model); in the same way, by having a more elaborated understanding about food additives (feature that integrates the dimension Cooking Skills, based on the top of the model) the consumer may demonstrate a greater awareness for industry's strategy to use "Flavour Intensifiers for Consumers' Loyalty" (belonging to the Factor Industry, placed at the bottom of the model).

Aiming to integrate wider and heterogeneous food systems-related surrounding contexts within the food literacy conceptualisation, the presence of Influential Factors within FOODLIT-PRO's Food Literacy Wheel 
intends to broaden the prospect of developing further food literacy-related actions integrating multistakeholders and multilevel approaches. That is, involving features from these factors - such as nutritional language (Nutritional), health behaviour change (Psychological), health-related consequences (Health), professional support (Learning Contexts), inter-sectorial policies (Policy), marketing's and social media's influence (Industry), circular economy (Sustainability), food-related trends and cultural representations (Social and Cultural) - embedded in food literacyrelated actions, aiming to tackle broader challenges regarding global sustainability across food systems.

\section{Conclusions}

With a mixed analysis, this study determined the structure for the definition of food literacy using a quantitative approach posteriorly on results previously obtained through an in-depth qualitative methodology. Additionally, this paper introduces a newly and groundbreaking food literacy framework that innovates by unifying definition, determinants and factors of influence - which advocates for multilevel and multi-stakeholder inclusion to provide for food systems integration and global sustainability.

Providing for food-related expert's perspectives, having a sample of both men and women from diverse action fields supplying for valuable manifested content is a strength of this study. As most of the interviews were personally conducted, nonverbal responses and its additional exploration was allowed by the interviewer's presence and interaction. Regarding the structure that a Delphi study can provide, the absence of this methodology is viewed as a limitation of this research. However, the use of a quantitative approach to explore the previously analysed qualitative content identified as food literacy definition highlights the innovative character of this study.

Within the field of food literacy, this study contributes not only to a significant shift on the understanding of this concept but also provides for essential insight to assist the development of food literacy instruments and interventions that aim for the change of attitudes, knowledge, and behaviours. This research also emphasises the importance of considering further psychological aspects on food literacy-related interventions.

Finally, at a broader spectrum of action regarding food systems, this FOODLIT-PRO's study hopes to inform the development of multi-sectorial and top- down governmental, commercial, and health-related regulations aiming for the improvement of food relations, behaviours and lifetime habits at the level of communities and nations.

\section{Acknowledgements}

The authors gratefully acknowledge the technical support provided by colleagues for the interviews' transcriptions, and the help provided with the graphic illustration by the colleagues from Audiovisual and Multimedia Unit from ISPA-Instituto Universitário.

\section{Disclosure statement}

No potential conflict of interest was reported by the author(s).

\section{Funding}

The WJCR-William James Center for Research is funded by FCT-Fundação para a Ciência e a Tecnologia (UID/PSI/ 04810/2019). This research was funded by FCT-Fundação para a Ciência e a Tecnologia (grant number SFRH/BD/ 128528/2017).

\section{References}

Amouzandeh C, Fingland D, Vidgen HA. 2019. A scoping review of the validity, reliability and conceptual alignment of food literacy measures for adults. Nutrients. 11(4):801.

Barbosa C, Pimenta P, Real H. 2017. Roda da Alimentação Mediterrânica e Pirâmide da Dieta Mediterrânica: comparação entre os dois guias alimentares. Acta Port Nutr. 11: 6-14.

Barnes M, Chan-Halbrendt C, Zhang Q, Abejon N. 2011. Consumer preference and willingness to pay for nonplastic food containers in honolulu, USA. JEP. 2(9): 1264-1273.

Begley A, Paynter E, Dhaliwal S. 2018. Evaluation tool development for food literacy programs. Nutrients. 10(11):1617.

Bellotti B. 2010. Food literacy: reconnecting the city with the country. Agric Sci. 2(3):29-34.

Bhunnoo R. 2019. The need for a food-systems approach to policy making. The Lancet. 393(10176):1097-1098.

Block LG, Grier SA, Childers TL, Davis B, Ebert JEJ, Kumanyika S, Laczniak RN, Machin JE, Motley CM, Peracchio L, et al. 2011. From nutrients to nurturance: a conceptual introduction to food well-being. J Public Policy Mark. 30(1):5-13.

Butcher LM, O'Sullivan TA, Ryan MM, Lo J, Devine A. 2019. Utilising a multi-item questionnaire to assess household food security in Australia. Health Promot J Austral. 30(1):9-17. 
Canfora I. 2016. Is the short food supply chain an efficient solution for sustainability in food market? Agric Agric Sci Procedia. 8:402-407.

Cohen J. 1960. A coefficient of agreement for nominal scales. Educ Psychol Meas. 20(1):37-46.

Costa PS, Santos NC, Cunha P, Cotter J, Sousa N. 2013. The use of multiple correspondence analysis to explore associations between categories of qualitative variables in healthy ageing. J Aging Res. 2013:1-12.

Cullen T, Hatch J, Martin W, Higgins JW, Sheppard R. 2015. Food literacy: definition and framework for action. Can J Diet Pract Res. 76(3):140-145.

Davis FB. 1964. Educational measurements and their interpretation. Belmont (CA): Wadsworth Publishing Co.

Desjardins E, Azevedo E, Davidson L, Samra R, Dunbar J, Thomas H, Ann Munoz M, King B, Maxwell T, WongMcGraw P. 2013. Making something out of nothing: food literacy among youth, young pregnant women and young parents who are at risk for poor health. In Locally Driven Collaborative Project Food Skills. Ontario (Canada): Public Health Ontario.

Di Franco G. 2016. Multiple correspondence analysis: one only or several techniques? Qual Quant. 50(3):1299-1315.

Durmus H, Balci E, Oral B, Sonkaya ZI. 2018. Knowledge of food literacy and food safety among turkish adults. Erciyes Med J. 40(2):81-86.

Elo S, Kyngäs H. 2008. The qualitative content analysis process. J Adv Nurs. 62(1):107-115.

[FAO] Food and Agriculture Organization of the United Nations, [IFAD] International Fund for Agricultural Development, [UNICEF] United Nations Children's Fund, [WFP] World Food Programme, [WHO] World Health Organization. 2019. The state of food security and nutrition in the world 2019. Safeguarding against economic slowdowns and downturns. Rome (Italy): FAO.

[FAO and WHO] Food and Agriculture Organization of the United Nations and World Health Organization. 2019. Food safety, everyone's business. Available from: http:// www.fao.org/3/ca4449en/ca4449en.pdf.

[FAO] Food and Agriculture Organization of the United Nations. 2017.The Future of food and agriculture: Trends and challenges. Rome (Italy): FAO.

[FAO] Food and Agriculture Organization of the United Nations. 2019. Framework for the urban food agenda: leveraging sub-national and local government action to ensure sustainable food systems and improved nutrition. Rome (Italy): FAO.

Food additives [WHO]. 2018. [Internet]. Jan 31. World Health Organization; [accessed 2020 Apr 13]. Available from: https://www.who.int/news-room/fact-sheets/detail/ food-additives.

Food safety. 2019. [Internet]. Jun 4. [WHO] World Health Organization; [accessed 2020 Feb 13]. Available from: https://www.who.int/news-room/fact-sheets/detail/foodsafety.

Global Panel on Agriculture and Food Systems for Nutrition. 2016. Food systems and diets: facing the challenges of the 21st century. London (UK): Global Panel.

Hsieh H-F, Shannon SE. 2005. Three approaches to qualitative content analysis. Qual Health Res. 15(9):1277-1288.
International Food Policy Research Institute. 2017. 2017 Global food policy report. Washington (DC): International Food Policy Research Institute.

Johnson RA, Wichern DW. 2007. Applied multivariate correspondence analysis. 6th ed. Upper Saddle River (NJ): Prentice-Hall.

Krause C, Sommerhalder K, Beer-Borst S, Abel T. 2016. Just a subtle difference? Findings from a systematic review on definitions of nutrition literacy and food literacy. Health Promot Int. 33(3):378-389.

Lindh H, Olsson A, Williams H. 2016. Consumer perceptions of food packaging: contributing to or counteracting environmentally sustainable development?: Consumer Perceptions of Food Packaging. Packag Technol Sci. 29(1):3-23.

Macdiarmid JI. 2014. Seasonality and dietary requirements: will eating seasonal food contribute to health and environmental sustainability? Proc Nutr Soc. 73(3):368-375.

Martínez-Hernández GB, Artés-Hernández F, Colares-Souza F, Gómez PA, García-Gómez P, Artés F. 2013. Innovative cooking techniques for improving the overall quality of a kailan-hybrid broccoli. Food Bioprocess Technol. 6(8): 2135-2149.

McHugh ML. 2012. Interrater reliability: the kappa statistic. Biochem Med. 22(3):276-282.

Medina FX. 2011. Food consumption and civil society: Mediterranean diet as a sustainable resource for the Mediterranean area. Public Health Nutr. 14(12A): 2346-2349.

Merriam SB. 2005. Adult learning. In: English LM, editor. International encyclopedia of adult education. New York (NY): Palgrave Macmillan. p. 42-48.

Mukhopadhyay S, Ukuku DO, Juneja VK, Nayak B, Olanya M. 2017. Principles of food preservation. In: Juneja V, Dwivedi H, Sofos J, editor. Microbial Control and food preservation. Food microbiology and food safety. New York (NY): Springer. p. 17-40.

Murimi MW. 2013. Healthy Literacy, Nutrition Education, and Food Literacy. J Nutrit Educ and Behav. 45(3):195.

National Academies of Sciences, Engineering, and Medicine. 2016. Food literacy: How do communications and marketing impact consumer knowledge, skills, and behavior? Workshop summary [Internet]. Pray L, editor. Washington (DC): National Academies Press; [accessed 2020 Feb 14]. Available from: http://www.nap.edu/cata$\log / 21897$.

Palumbo R. 2016. Sustainability of well-being through literacy. The effects of food literacy on sustainability of wellbeing. Agric Agric Sci Procedia. 8:99-106.

Palumbo R, Adinolfi P, Annarumma C, Catinello G, Tonelli M, Troiano E, Vezzosi S, Manna R. 2019. Unravelling the food literacy puzzle: evidence from Italy. Food Policy. 83: 104-115.

Pendergast D, Garvis S, Kanasa H. 2011. Insight from the public on home economics and formal food literacy. Fam Consum Sci Res J. 39(4):415-430.

Perry EA, Thomas H, Samra HR, Edmonstone S, Davidson L, Faulkner A, Petermann L, Manafò E, Kirkpatrick SI. 2017. Identifying attributes of food literacy: a scoping review. Public Health Nutr. 20(13):2406-2415.

Poelman MP, Dijkstra SC, Sponselee H, Kamphuis CBM, Battjes-Fries MCE, Gillebaart M, Seidell JC. 2018. 
Towards the measurement of food literacy with respect to healthy eating: the development and validation of the self perceived food literacy scale among an adult sample in the Netherlands. Int J Behav Nutr Phys Act. 15(1):54.

Powell LJ, Wittman H. 2018. Farm to school in British Columbia: mobilizing food literacy for food sovereignty. Agric Hum Values. 35(1):193-206.

Roncero-Ramos I, Mendiola-Lanao M, Pérez-Clavijo M, Delgado-Andrade C. 2017. Effect of different cooking methods on nutritional value and antioxidant activity of cultivated mushrooms. Int J Food Sci Nutr. 68(3): 287-297.

Ronto R, Ball L, Pendergast D, Harris N. 2016. Adolescents' perspectives on food literacy and its impact on their dietary behaviours. Appetite. 107:549-557.

Rosas R, Pimenta F, Leal I, Schwarzer R. 2019. FOODLITPRO: food literacy domains, influential factors and determinants-a qualitative study. Nutrients. 12(1):88.

Santarossa S, Ciccone J, Woodruff SJ. 2015. An evaluation of the Kinect-Ed presentation, a motivating nutrition and cooking intervention for young adolescents in grades 6-8. Appl Physiol Nutr Metab. 40(9):945-950.

Schwarzer R, Luszczynska A. 2015. Health action process approach. In: Mark Conner and Paul Norman, editor. Predicting and changing health behaviour: research and practice with social cognition models. 3rd ed. England: Open University Press. p. 252-278.

Slater J, Falkenberg T, Rutherford J, Colatruglio S. 2018. Food literacy competencies: a conceptual framework for youth transitioning to adulthood. Int J Consum Stud. 42(5):547-556.

Slater JJ, Mudryj AN. 2016. Self-Perceived eating habits and food skills of canadians. J Nutrit Educ Behav. 48(7): 486-495.e1.

Sumner J. 2013. Food literacy and adult education: learning to read the world by eating. Can J Study Adult Educ. 25(2):79-92.

The World Bank. 2019. GDP growth (annual \%). Washington (DC): World Bank Group.

Thomas H, Azevedo Perry E, Slack J, Samra HR, Manowiec E, Petermann L, Manafò E, Kirkpatrick SI. 2019. Complexities in conceptualizing and measuring food literacy. J Acad Nutr Diet. 119(4):563-573.
Thomas HM, Irwin JD. 2011. Cook It Up! A communitybased cooking program for at-risk youth: overview of a food literacy intervention. BMC Res Notes. 4(1):495.

Truman E, Lane D, Elliott C. 2017. Defining food literacy: a scoping review. Appetite. 116:365-371.

Uhlmann K, Lin B, Ross H. 2018. Who Cares? The importance of emotional connections with nature to ensure food security and wellbeing in cities. Sustainability. 10(6): 1844.

Vaitkeviciute R, Ball LE, Harris N. 2015. The relationship between food literacy and dietary intake in adolescents: a systematic review. Public Health Nutr. 18(4):649-658.

van Dooren C, Marinussen M, Blonk H, Aiking $H$, Vellinga P. 2014. Exploring dietary guidelines based on ecological and nutritional values: a comparison of six dietary patterns. Food Policy. 44:36-46.

Velardo S. 2015. The nuances of health literacy, nutrition literacy, and food literacy. J Nutr Educ Behav. 47(4): 385-389.e1.

Vettori V, Lorini C, Milani C, Bonaccorsi G. 2019. Towards the Implementation of a conceptual framework of food and nutrition literacy: providing healthy eating for the population. Int J Environ Res Public Health. 16(24):5041.

Vidgen HA, Gallegos D. 2014. Defining food literacy and its components. Appetite. 76:50-59.

Warrens MJ. 2014. New interpretations of Cohen's Kappa. J Math. 2014:1-9.

Wickham CA, Carbone ET. 2018. What's technology cooking up? A systematic review of the use of technology in adolescent food literacy programs. Appetite. 125:333-344.

Wijayaratne SP, Reid M, Westberg K, Worsley A, Mavondo F. 2018. Food literacy, healthy eating barriers and household diet. Eur J Mark. 52(12):2449-2477.

Wilkins JL. 2002. Consumer perceptions of seasonal and local foods: a study in a U.S. community. Ecology of Food and Nutrition. 41(5):415-439.

Willett W, Rockström J, Loken B, Springmann M, Lang T, Vermeulen S, Garnett T, Tilman D, DeClerck F, Wood $A$, et al. 2019. Food in the anthropocene: the EAT-Lancet Commission on healthy diets from sustainable food systems. The Lancet. 393(10170):447-492. 